\title{
EFECTO DE LA LABRANZA MÍNIMA Y LA CONVENCIONAL EN ARROZ (Oryza sativa L.) EN LA REGIÓN HUETAR NORTE DE COSTA RICA ${ }^{1}$
}

\author{
Luis A. Rojas², Alexánder Mora ${ }^{3}$, Harold Rodríguez ${ }^{3}$
}

\section{RESUMEN}

Efecto de la labranza mínima y de la labranza convencional en arroz (Oryza sativa L.) en la Región Huetar Norte de Costa Rica. En la zona norte de Costa Rica en donde se establecieron dos lotes: uno de labranza mínima (3 ha), y otro de labranza convencional ( 0,80 ha). La siembra en ambos sistemas de labranza se realizó con una sembradora abonadora hidráulica. La variedad de arroz utilizada fue la CR 2515. En labranza mínima la siembra se realizó en condiciones de alta humedad, labor que prácticamente es imposible en labranza convencional. En este mismo sistema de labranza hubo una menor germinación y población de plantas de arroz, principalmente en donde había más rastrojo. En el ciclo 2001 en labranza mínima hubo mayor densidad de Murdania nudiflora, Echinocloa colonum y Digitaria sp. En labranza convencional la maleza predominante fue Cyperus rotundus. Las plagas y enfermedades no fueron factor limitante en el manejo del cultivo en ambos sistema de labranza, no obstante, en labranza mínima se observó una mayor población de hormigas del género Solenopsis. En el ciclo de siembra 2001 en labranza convencional hubo mayor número de panículas $/ \mathrm{m}^{2}>$ a 20 cm que en labranza mínima, mientras que en el ciclo 2000 en labranza mínima la cantidad de panículas $/ \mathrm{m}^{2}>20 \mathrm{~cm}$ fue muy baja. De igual forma, en labranza convencional el número de granos por panícula fue mayor en todas las longitudes de panículas y el peso de 1000 granos fue similar a labranza mínima en todas las longitudes de panículas. El rendimiento estimado fue mayor en labranza convencional con 6,74 t/ha, mientras que en labranza mínima fue de 5,91 t/ha.

\begin{abstract}
Effect of minimun and conventional tillage in rice (Oryza sativa L.) in the Northern Huetar Region in Costa Rica. In the north area of Costa Rica two plots were sown: one of minimum tillage ( $3 \mathrm{ha}$ ), and another of conventional tillage ( 0,80 ha). Both tillage systems were planted with a hydraulic sower fertilizer. The variety of rice used was the CR 2515 . In minimum tillage the sow work was conducted under conditions of high humidity, job that is practically impossible under conventional tillage. Under this same tillage system, there was a lower germination and population of rice plants, mainly in the areas where there was more stubble. In the planting cycle 2001, the minimum tillage had larger density of Murdania nudiflora, Echinocloa colonum and Digitaria sp. In conventional tillage the most prevailing weed was Cyperus rotundus. The plagues and diseases were not a limiting factor in the handling of the crop in both tillage systems, however, in the minimum tillage there were a largger incidence of ants, of the genus Solenopsis. In the sow 2001 cycle, in conventional tillage had larger number of panicles $/ \mathrm{m}^{2}>$ to $20 \mathrm{~cm}$ that in minimum tillage, while in the 2000 cycle in minimum tillage the quantity of panicles $/ \mathrm{m}^{2}>20 \mathrm{~cm}$ was very low. Like wise, the number of grains per panicle was larger for minimum tillage in all the panicles lengths, and the weight of 1000 grains was similar to minimum tillage in all the panicles lengths. The estimated yield was higher in the system of conventional tillage with $6.74 \mathrm{t} / \mathrm{ha}$, while in minimum tillage it was of $5.91 \mathrm{t} / \mathrm{ha}$.
\end{abstract}

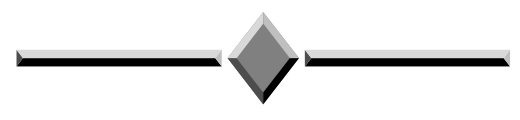

1 Recibido para publicación el 6 de agosto del 2002.

2 Instituto Tecnológico de Costa Rica, Sede Regional San Carlos. Tel (506) 475-50-33 ext 225 ó 216 Fax (506)475-53-95 e-mail: 1rojas@costarricense.cr

3 Escuela de Agronomía, Instituto Tecnológico de Costa Rica, Sede Regional San Carlos. 


\section{INTRODUCCIÓN}

En Costa Rica la producción de arroz ha venido en disminución, a consecuencia de las políticas mundiales de libre comercio, en donde no se estimula al agricultor nacional sino que se importa arroz a un menor precio que el producido en nuestro país.

En los últimos años se viene hablando de conceptos como desarrollo sostenible y agricultura sostenible. Para lograr una mayor sostenibilidad en un sistema de cultivo, es necesario implementar un cambio en la tecnología de producción, de modo que se disminuyan los costos de producción o se aumente la productividad (Arauz 1996). Una forma de mejorar la tecnología de la producción en granos básicos, es mediante la no labranza del terreno para la siembra, conocida como labranza mínima, labranza de conservación, o siembra directa (García 2001, Gassen y Gassen 1996).

En general, al implementar el método de labranza mínima se logra, entre otros aspectos, reducir la erosión hídrica y eólica del suelo, hay mayor facilidad de siembra y de cosecha, menor compactación del suelo, menor consumo energético, se mejora las propiedades físico, químicas y biológicas del suelo, y se disminuye la incidencia de malezas anuales (Pitty 1997, Unger et al. 1995).

Dentro de un sistema productivo, la labranza mínima ofrece beneficios en el corto, mediano y largo plazo, no obstante, es poca la investigación desarrollada en nuestro país sobre esta tecnología. Al respecto, en las fincas que han implementado este sistema de labranza, existen dudas sobre los beneficios en el mediano y largo plazo, y sobre la aplicabilidad a diferentes sistemas de producción. En vista de ello se realizó este trabajo que es parte de un proyecto de investigación que pretende implementar el uso de la labranza mínima como alternativa de producción sostenible en granos básicos en la Región Huetar Norte.

El objetivo es evaluar el efecto de la labranza mínima y de la labranza convencional sobre el establecimiento, comportamiento fitosanitario y el rendimiento en dos ciclos de siembra del cultivo de arroz (Oryza sativa L.) en la Zona Norte de Costa Rica.

\section{MATERIALES Y MÉTODOS}

El trabajo se realizó en Finca La Vega del Instituto Tecnológico de Costa Rica, ubicado en Santa Clara de San Carlos, Alajuela, Costa Rica. Según Holdridge
(1987), esta zona se clasifica como bosque húmedo tropical.

El área experimental constó de 0,8 ha sembrado en labranza convencional y tres ha sembrado en labranza mínima. Este trabajo consideró dos ciclos de siembra: el ciclo 2000 sembrado en octubre, y el ciclo 2001 sembrado en mayo. En el ciclo 2000 sólo se sembró el área de labranza mínima debido a que en labranza convencional la alta precipitación impidió la entrada de la sembradora. Esto no sucedió en labranza mínima.

La variedad de arroz utilizada fue la CR2515 sembrada a una densidad de $134 \mathrm{~kg} / \mathrm{ha}$. La siembra en ambos sistemas de labranza se realizó con una sembradora de labranza mínima Tatú modelo SDA2E de 15 líneas de descarga de semilla y fertilizante, con un ancho de labor de 2,4 m y una capacidad de depósito de semilla de $430 \mathrm{~kg}$ y de $680 \mathrm{~kg}$ de fertilizante.

Las variables evaluadas fueron: población de plantas de arroz evaluada antes del macollamiento a los 15 días después de la siembra (dds), y después del macollamiento a los $60 \mathrm{dds}$, densidad de malezas evaluada previo a la primera aplicación de herbicida posemergente mediante muestreos con cuadrícula de $0,25 \mathrm{~m}^{2}$, incidencia de plagas y enfermedades, y los factores de rendimiento (número de panículas $/ \mathrm{m}^{2}$, número de granos por panícula, peso de 1000 granos, y el rendimiento estimado). En la evaluación de los factores de rendimiento, excepto el rendimiento estimado, las panículas se clasificaron en tres tamaños: $<15 \mathrm{~cm}, 15$ a $20 \mathrm{~cm}$, y $>$ a $20 \mathrm{~cm}$.

En relación con el rendimiento se hizo una estimación del mismo en vista que no fue posible obtener el rendimiento real dado por la arrocera por razones de manejo de la cosecha del área experimental. La estimación se obtuvo con base en el número de panículas por hectárea, asociado al tamaño de las panículas y al número de granos por panícula. Luego con el peso promedio de 1.000 granos se extrapoló el peso total de todos los granos. Este peso total se multiplicó por 0,7 ya que se estima una pérdida de cosecha de un $30 \%$.

En el ciclo 2000 no se evaluó el número de granos/ panícula.

\section{RESULTADOS Y DISCUSIÓN}

\section{Población de plantas de arroz}

En el ciclo 2001 en el sistema de labranza mínima se obtuvo un promedio de $197 \pm 9$ tallos $/ \mathrm{m}^{2}$, antes del 
macollamiento, mientras que en labranza convencional el promedio fue de $251 \pm 15$ plantas $/ \mathrm{m}^{2}$. Por otra parte, después del macollamiento se obtuvo $568 \pm 10$ y $661 \pm$ 24 plantas $/ \mathrm{m}^{2}$ en labranza mínima y en labranza convencional, respectivamente (Figura 1 ).

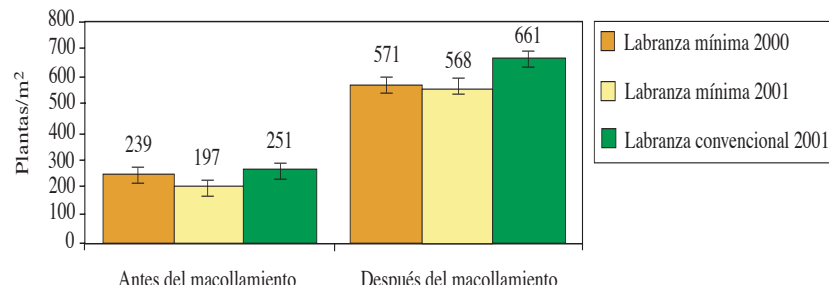

Epoca de muestreo

Figura 1. Población de plantas de arroz antes y después del macollamiento. Finca la Vega, ITCR, San Carlos, Costa Rica. 2001.

Se observa que la población de plantas en labranza mínima en ambos ciclos de cultivo fue menor en comparación con labranza convencional. Esta disminución probablemente fue causada por la cantidad de rastrojo que había en el suelo durante la siembra (Figura 2) que se constituyó en una barrera física para una buena germinación y desarrollo inicial; de igual forma, pudo haber influido el exceso de humedad del suelo el cual tiende a ser mayor en un sistema de labranza mínima.

Por lo anterior, en un sistema de labranza mínima es recomendable utilizar una densidad de siembra mayor para contrarrestar el efecto de una menor población de plantas por la pérdida de plantas o de semillas como consecuencia del rastrojo.

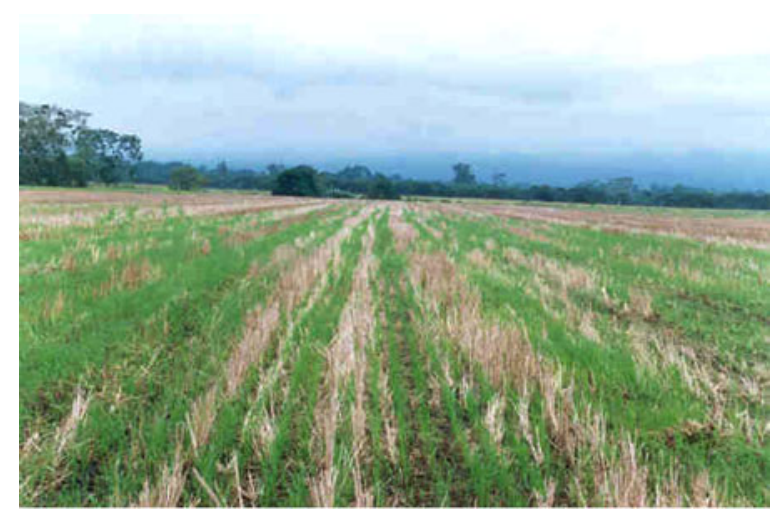

Figura 2. Población de plantas de arroz obtenida en labranza mínima al inicio del macollamiento. Finca la Vega, ITCR, San Carlos, Costa Rica. 2001.

\section{Densidad de malezas}

En el sistema de labranza mínima la especie Cyperus rotundus tuvo una densidad de 10,2 plantas por cuadrícula $\left(0,25 \mathrm{~m}^{2}\right)$ en el primer ciclo del 2000 y en el ciclo 2001 disminuyó a 3,2 plantas. Por lo anterior, fue evidente que este sistema de labranza no favoreció el desarrollo de esta maleza la cual se observó bien establecida en el sistema de labranza convencional (Figura 3) durante el primer ciclo del 2001 donde tuvo una densidad de 41,2 plantas/cuadrícula. Se debe recalcar que en esta finca precisamente la mecanización continua ha sido la principal causante de la propagación de esta maleza por los lotes de siembra.

Por el contrario, en el sistema de labranza mínima la especie Murdania nudiflora en el ciclo de siembra 2000 tuvo una densidad de 2,3 plantas/cuadrícula, no obstante, en el ciclo 2001 aumentó a 17, 5 plantas/cuadrícula (Figura 4). Se debe aclarar que, independientemente del sistema de labranza, la mayor incidencia de ésta y otras malezas se da al inicio de las lluvias (mayo). En este caso particular, la siembra del 2000 correspondió al segundo ciclo de siembra (octubre) y la siembra del 2001 correspondió al primer ciclo de siembra (mayo).

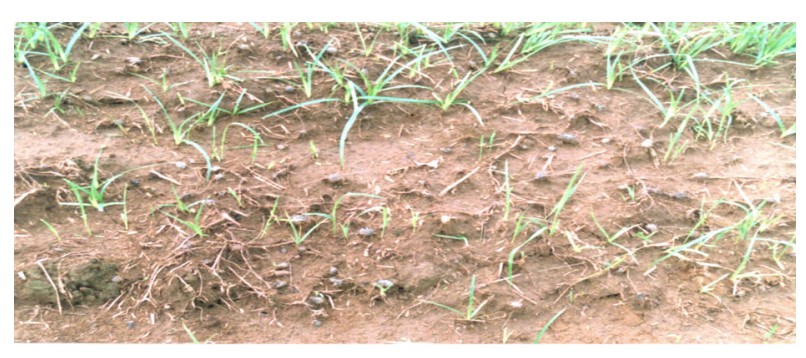

Figura 3. Población de coyolillo (Cyperus rotundus) en labranza convencional en arroz. Finca La Vega, ITCR, San Carlos, Costa Rica. 2001.

\section{Manejo de plagas y enfermedades}

En ambos ciclos de cultivo en general no hubo problemas importantes de plagas y enfermedades. Se debe destacar que se observó la presencia de una alta población de hormigas del género Solenopsis, especialmente en el área de labranza mínima donde hubo una mayor presencia de rastrojo. Estas hormigas se comen la raíz de la planta de arroz conforme ésta va germinando. Por tal motivo, se convierte en una limitante el uso de la cobertura o del rastrojo en la labranza mínima ya que permite el establecimiento y desarrollo de colonias de hormigas. 


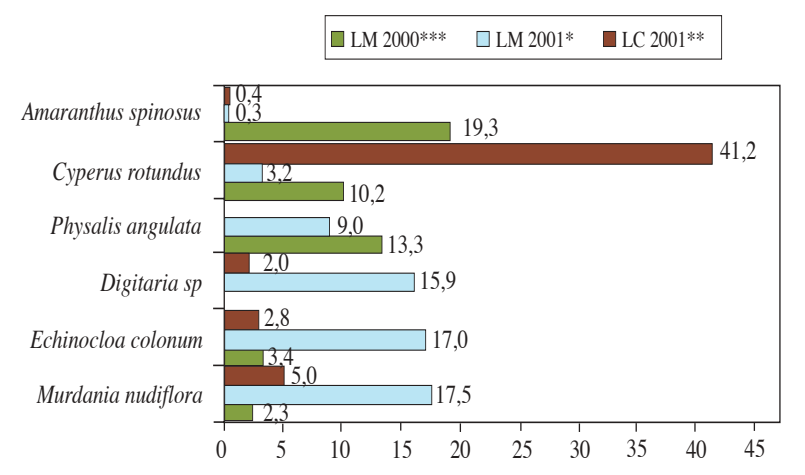

* Labranza mínima I ciclo 2001

** Labranza convencional I ciclo 2001

*** Labranza mínima II ciclo 2000

Figura 4. Densidad de malezas obtenida en dos ciclos de siembra de arroz en labranza mínima y en labranza convencional. Finca La Vega, ITCR, San Carlos, Costa Rica. 2001.

Por otra parte, la variedad CR2515 es susceptible a Rhyzoctonia, por lo que se hicieron dos evaluaciones (60 y 90 días después de la siembra) en ambos sistemas de labranza. El primero se llevó a cabo antes de la primera aplicación del fungicida Amistar 50 WG (Azoxistrobina), y el segundo posterior a esta aplicación.

En el sistema de labranza mínima se observó un porcentaje de incidencia de Rhyzoctonia apenas ligeramente superior $(13,9 \pm 8,07 \%)$ en comparación con la incidencia obtenida en labranza convencional $(12,07 \pm$ 10,07) (Figura 6).

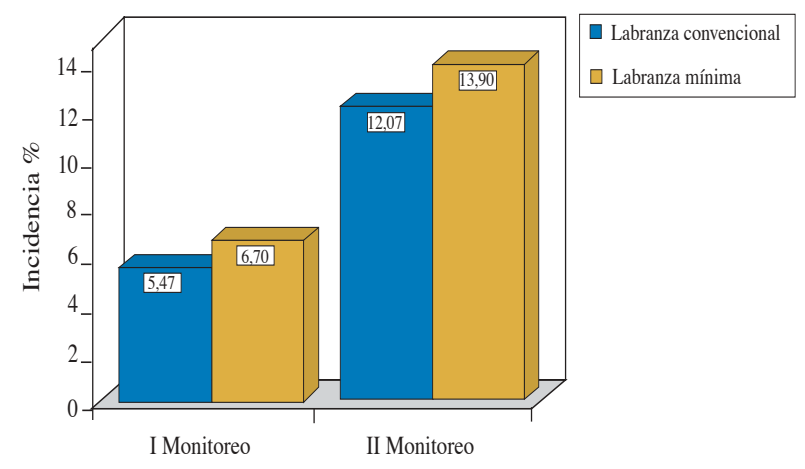

Figura 5. Incidencia (\%) de añublo de la vaina (Rhizoctonia solani) obtenida en el cultivo de arroz sembrado en labranza mínima y en labranza convencional. Finca LaVega, ITCR, San Carlos, Costa Rica. 2001.

\section{Factores de rendimiento}

Al comparar el número de panículas $/ \mathrm{m}^{2}$ de acuerdo a los rangos de longitud en ambos ciclos de cultivo, se observó que en el ciclo 2001, en ambos sistemas de labranza, la mayor cantidad de panículas se obtuvo en el tamaño > $20 \mathrm{~cm}$ con 266,80 panículas en labranza convencional y 228,52 panículas en labranza mínima, mientras que en el ciclo 2000 en labranza mínima apenas se obtuvieron 53,53 panículas $/ \mathrm{m}^{2}$. Esta diferencia de un ciclo de siembra a otro se debe a que el arroz sembrado en el ciclo 2000 fue muy afectado por el ganado, además que en la segunda siembra hubo menos humedad. Estas condiciones favorecen un menor crecimiento de la planta y por ende el tamaño de la panícula. En consecuencia, al haber menos panículas con tamaño $>20 \mathrm{~cm}$ habrá mayor cantidad de panículas con longitudes inferiores y por lo tanto habrá también un menor rendimiento.

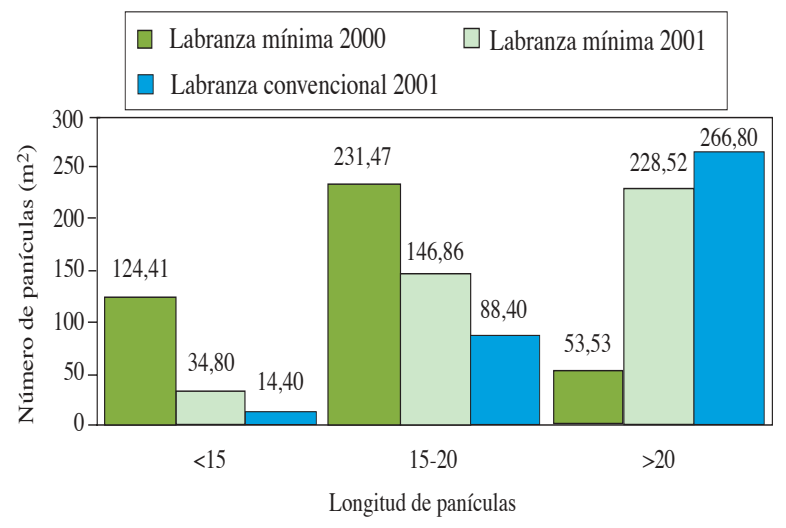

Figura 6. Número de panículas $/ \mathrm{m}^{2}$ obtenida de acuerdo al tamaño de panículas, en dos ciclos de siembra y en dos sistemas de labranza. Finca La Vega, ITCR, San Carlos, Costa Rica. 2001.

En el sistema de labranza mínima el número de granos por panícula $>20 \mathrm{~cm}$ fue de $95,74 \pm 20,93$ granos, mientras que en labranza convencional fue de $110,96 \pm 30,20$ granos. Por otra parte, en el rango de 15 a $20 \mathrm{~cm}$ en labranza mínima se obtuvo $56 \pm 13$,30 granos/panícula, en tanto que en labranza convencional se obtuvo $72,49 \pm 23,45$ granos/panícula. Por último, en la frecuencia de longitud de panículas < a $15 \mathrm{~cm}$ el número de granos/panícula fue de 20,40 \pm 7,9 y $26 \pm 7,07$ en la labranza mínima y labranza convencional, respectivamente (Figura 7). 


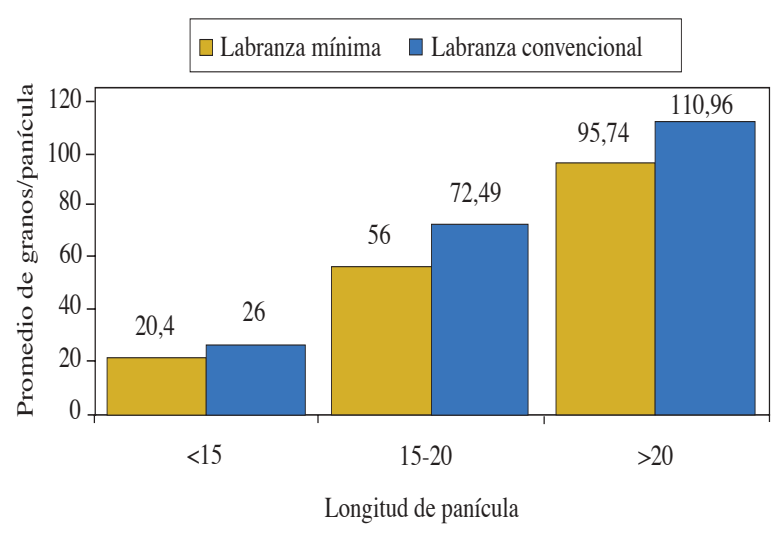

Figura 7. Número de granos / panícula de arroz sembrado en labranza mínima y en labranza convencional. Finca La Vega, ITCR, San Carlos, Costa Rica. 2001.

El peso de 1000 granos en el sistema de labranza mínima tuvo una variación del ciclo de siembra del año 2000 al 2001. Respecto al ciclo 2000 , se obtuvo un promedio de peso de $18,14 \mathrm{~g}$ en los tres rangos de longitud de panícula $(<15,15-20$ y $>20 \mathrm{~cm})$, mientras que en el ciclo 2001 este alcanzó mayor peso en panículas superiores a $20 \mathrm{~cm}$ con $27,84 \mathrm{~g}$ y el menor peso fue de $26,34 \mathrm{~g}$ en panículas entre 15 y $20 \mathrm{~cm}$ de longitud. Por otra parte, en el sistema de labranza convencional el peso de 1000 granos fue de $27,29 \mathrm{~g}$ en panículas > a 20 $\mathrm{cm}$, de $22,7 \mathrm{~g}$ en panículas de $15-20 \mathrm{~cm}$, y de $25,96 \mathrm{~g}$ en panículas < a $15 \mathrm{~cm}$ de longitud (Figura 8).

El rendimiento fue mayor en el sistema de labranza convencional con $6,74 \mathrm{t} / \mathrm{ha}$, mientras que en labran-

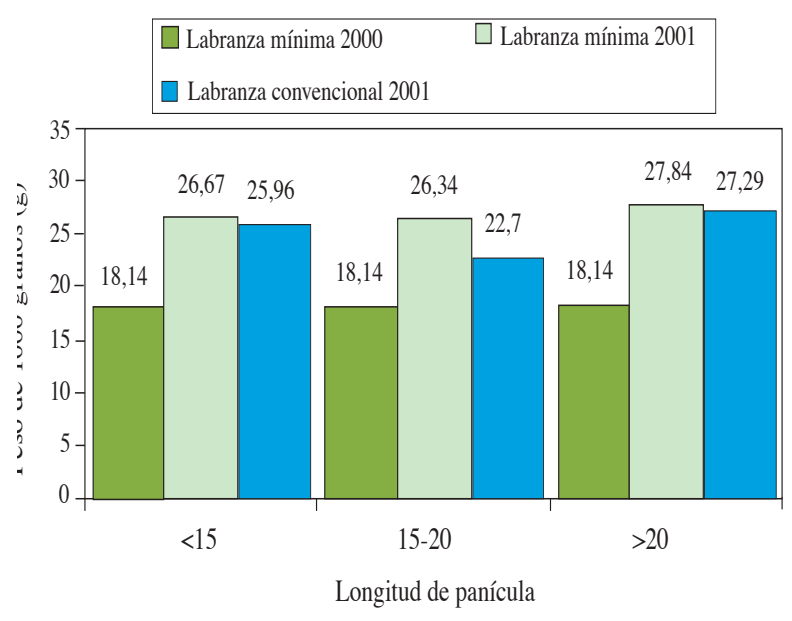

Figura 8. Peso de 1000 granos obtenidos en dos ciclos de arroz sembrado en labranza mínima y en labranza convencional. Finca La Vega, ITCR, San Carlos. 2001. za mínima fue de 5,91 t/ha (Figura 9). Estos resultados coinciden con los factores de rendimiento, así como también con la población de plantas evaluadas antes y después del macollamiento, en las que el sistema de labranza convencional tuvo una mejor respuesta. Esto no quiere decir que labranza mínima no sea una alternativa sino que se deben adecuar las densidades de siembra para así tener una mayor población de plantas que proporcione un mejor rendimiento.

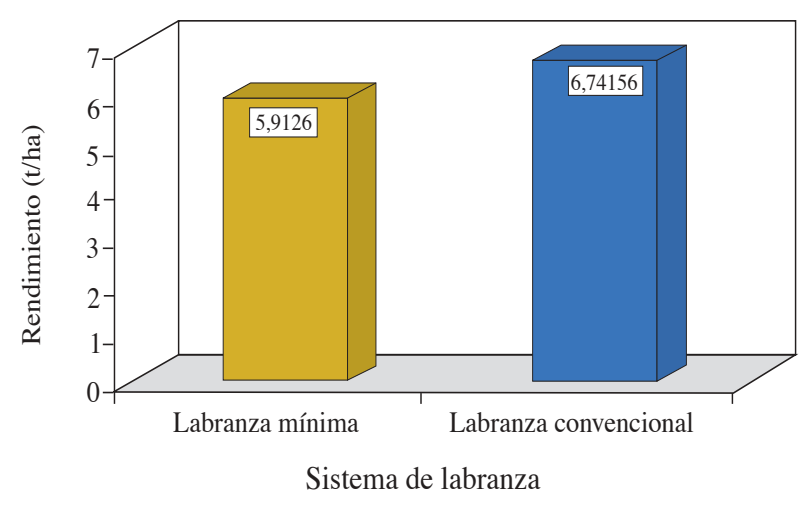

Figura 9. Rendimiento estimado (t/ha) estimado de arroz sembrado en labranza mínima y en labranza convencional. Finca La Vega, ITCR, San Carlos, Costa Rica. 2001.

\section{CONCLUSIONES}

La siembra en labranza mínima se realizó en condiciones de alta humedad, labor que sería muy difícil en labranza convencional.

La población de plantas en labranza mínima en ambos ciclos de cultivo fue menor en comparación con la labranza convencional, debido probablemente a la cantidad de rastrojo que había en el suelo que ejerce una barrera física para la germinación y desarrollo inicial del cultivo.

En el ciclo 2001 en labranza mínima hubo mayor densidad de Murdania nudiflora, Echinocloa colonum y Digitaria sp. en comparación con labranza convencional y con labranza mínima del ciclo 2000. En el sistema de labranza convencional la maleza de mayor presencia fue Cyperus rotundus.

En ambos ciclos de cultivo las plagas y enfermedades no fueron factor limitante en el manejo del cultivo 
en ambos sistema de labranza, sin embargo, se observó una alta población de hormigas del género Solenopsis, en labranza mínima. La incidencia de Rhyzoctonia solani se consideró baja en ambos sistemas de labranza.

En el ciclo de siembra 2001 en labranza convencional se obtuvo más panículas $/ \mathrm{m}^{2}>$ a $20 \mathrm{~cm}$ que en labranza mínima, mientras que en el ciclo 2000 sembrado en labranza mínima la cantidad de panículas $/ \mathrm{m}^{2}>20$ cm fue muy baja comparado con el ciclo 2001.

En el ciclo de siembra 2001 en labranza convencional el número de granos por panícula fue mayor en todos los tamaños de panículas comparado con labranza mínima.

En el ciclo de siembra 2001 tanto en labranza convencional como en labranza mínima el peso de 1000 granos fue similar en todos los rangos de longitudes de panículas, no obstante, en el ciclo 2000 sembrado en labranza mínima el peso fue muy inferior.

El rendimiento estimado fue superior en el sistema de labranza convencional.

\section{LITERATURA CITADA}

ARAUZ, L. 1996. La protección de cultivos en la agricultura sostenible: perspectivas para Costa Rica. Manejo Integrado de Plagas (CR) 41:29-36.

GARCÍA, F. 2001. Ventajas de la siembra directa. (en línea). Santa Fe, Argentina. Consultado 23 en 2002. Disponible en http:/www.ecampo.com/sections/news/archive.php?CatUuid=91D0DF04\%2DE269\%2D11D3\%2DA5140006292E2740\&.

GASSEN, D; GASSEN, F. 1996. Plantio direto o camino do futuro. Brasil. A Idea Sul Editora. 207 p.

HOLDRIDGE, L. 1987. Ecología basada en zonas de vida. San José, C.R., ICA. 216 p.

PITTY, A. 1997. Introducción a la biología, ecología y manejo de malezas. Tegucigalpa, Honduras. Zamorano. 300p.

UNGER, P, W; LARYEA, K. B.; STEWART, B. A. 1995. Criterios para la selección de sistemas y prácticas de labranza. In: Reunión Bienal de la Red Latinoamericana de Labranza Conservacionista (20, 1993, Guanare, Acarigua, Venezuela, VE, FONAIAP. p.118-146. 